\title{
Essais
}

Revue interdisciplinaire d'Humanités

\section{Dynamiques autour de la production de fleurs coupées en Île-de-France}

Dynamics of cut flower production in île-de-France

\section{Léa Benoit}

\section{(2) OpenEdition}

\section{Journals}

Édition électronique

URL : https://journals.openedition.org/essais/7353

DOI : 10.4000/essais.7353

ISSN : 2276-0970

Éditeur

École doctorale Montaigne Humanités

\section{Édition imprimée}

Date de publication : 1 mars 2021

Pagination : 47-63

ISBN : 978-2-492780-00-4

ISSN : $2417-4211$

\section{Référence électronique}

Léa Benoit, " Dynamiques autour de la production de fleurs coupées en Île-de-France », Essais [En ligne], Hors-série 6 | 2021, mis en ligne le 16 mars 2021, consulté le 18 janvier 2023. URL : http:// journals.openedition.org/essais/7353; DOl : https://doi.org/10.4000/essais.7353 


\section{Dynamiques autour de la production de fleurs coupées en Ile-de-France}

\section{Léa Benoit}

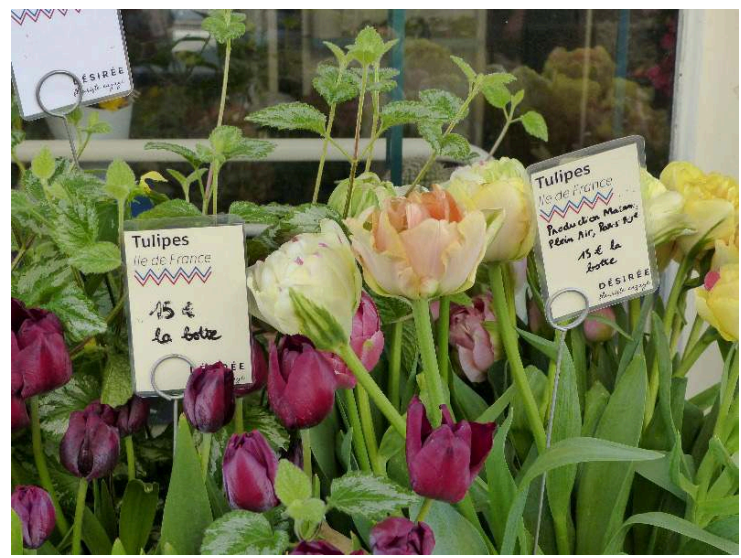

Figure 1 : Tulipes parisiennes chez un fleuriste parisien Source : Léa Benoit, avril 2019

Mardi 17 mars 2020, les Franciliens sont confinés, les fleuristes fermés, le commerce des fleurs coupées à l'arrêt. Cette immobilité cache cependant une autre réalité. Très vite, en Ile-de-France, plusieurs personnes s'activent pour soutenir les producteurs locaux, contraints de jeter leurs fleurs en pleine saison printanière. Des ventes solidaires par cagnottes virtuelles avec livraisons « sans contact » sont par exemple effectuées. Ces initiatives mettent sur le devant de la scène les producteurs franciliens rappelant ainsi que la production de fleurs coupées n'a pas totalement disparue dans la région malgré des statistiques alarmantes. Comme partout en France, le nombre de producteurs y a en effet drastiquement chuté. La production est aujourd'hui davantage l'affaire d'investisseurs qui cultivent des fleurs de manière intensive dans la zone intertropicale ${ }^{1}$. Il s'agit donc d'une agriculture déterritorialisée dominée par des

1 Léa Benoit, Bernard Calas, Sylvain Racaud, Olivier Ballesta, Lucie Drevet-Demettre, « Roses d'Afrique, roses du monde ", Géoconfluences, 2017. 
"stratégies planétaires des firmes » dont les produits sont plutôt «standardisés mais circulent physiquement à travers le monde $"^{2}$. Pourtant, un dynamisme nouveau semble à l'œuvre comme en témoigne l'installation de nouveaux producteurs en Ile-de-France, mais surtout l'émergence de modes de production et de commercialisation alternatifs. Ce paradoxe pourrait être interprété comme un processus de reterritorialisation de la production de fleurs coupées en Ile-de-France. Reterritorialisation et non territorialisation car il ne s'agit pas d'un retour en arrière mais plutôt d'une réintroduction de l'agriculture sur un territoire à l'aide de nouvelles pratiques de production et de commercialisation $^{3}$. Si tel est bien le cas, cela viendrait démontrer une fois de plus que la mondialisation est à la fois facteur d'homogénéisation à l'échelle mondiale et d'enracinement à l'échelle localé .

Pour comprendre les facteurs et enjeux de cette potentielle reterritorialisation, une enquête a été réalisée en 2019 et 2020. Plusieurs entretiens semi-directifs ont ainsi été conduits avec des producteurs, fleuristes, gérants de plateformes de commercialisation en ligne ou d'associations franciliennes (Figure 2). Pendant ces entretiens, il a été demandé aux différents interlocuteurs de raconter leur histoire, leurs motivations, leurs objectifs, les conditions dans lesquelles ils réalisent leurs activités (moyens financiers, techniques, fournisseurs, origine des variétés).

Sans prétendre être exhaustif, l'échantillonnage permet de refléter au mieux la diversité des activités franciliennes en termes de production et de commercialisation de fleurs coupées, des trajectoires et des stratégies d'intégration territoriale des acteurs concernés.

De plus, un travail de participation observante a été mené avec le Collectif de la fleur française. Ce Collectif est une association qui s'inspire du mouvement Slow Flowers des pays anglo-saxons et promeut la production et la commercialisation de fleurs locales et de saison. Plusieurs acteurs franciliens produisant et/ou commercialisant des fleurs coupées y adhèrent. L'association constitue donc une ressource pour l'enquête 5 .

2 Hervé Thery, «Mondialisation, déterritorialisation, reterritorialisation, Bulletin de l'Association de géographes français, 3, 2008, p. 324-331.

3 Laurent Rieutort, "Dynamiques rurales françaises et re-territorialisation de l'agriculture ", L'Information géographique, 1, 2009, p. 30-48.

4 Hervé Thery, «Mondialisation, déterritorialisation, reterritorialisation, Bulletin de l'Association de géographes français, 3, 2008, p. 324-331.

5 La participation observante a pu être menée dans le cadre d'entretiens réguliers entre 2018 et 2020 et d'un stage effectué fin 2019. L'objectif du stage était de contribuer à la structuration de l'association. Pour cela, trois documents ont été réalisés à la suite de la mise en place de questionnaires : un document sur les fournisseurs des adhérents, un calendrier des fleurs de saison, une charte qualité. 
Enfin, une analyse de données chiffrées concernant la production en Ile-deFrance a été réalisée malgré leurs limites (données trop anciennes et trop rares).

\begin{tabular}{|c|c|c|c|}
\hline & Nom de la structure & Localisation & Statut/activité(s) \\
\hline \multirow{8}{*}{$\begin{array}{l}\mathrm{P} \\
\mathrm{R} \\
\mathrm{O} \\
\mathrm{D} \\
\mathrm{U} \\
\mathrm{C} \\
\mathrm{T} \\
\mathrm{I} \\
\mathrm{O} \\
\mathrm{N}\end{array}$} & Les serres de Misery & Essonne & Exploitation agricole \\
\hline & Des roses dans mon jardin & Seine-et-Marne & Exploitation agricole \\
\hline & Flick & Seine-et-Marne & Exploitation agricole \\
\hline & Les fleurs du moulin & Seine-et-Marne & Exploitation agricole \\
\hline & Plein air & Paris & Ferme urbaine \\
\hline & Ferme florale urbaine & Paris & Ferme urbaine \\
\hline & Halage & Seine-St-Denis & Chantier d'insertion - urbain \\
\hline & Fleurs de Cocagne & Essonne & Chantier d'insertion - rural \\
\hline \multirow{6}{*}{$\begin{array}{l}\text { D } \\
\text { I } \\
\text { S } \\
\text { T } \\
\text { R } \\
\text { I } \\
\text { B } \\
\text { U } \\
\text { T } \\
\text { I } \\
\mathbf{O} \\
\mathbf{N}\end{array}$} & Désirée & Paris & Café-fleuriste en boutique \\
\hline & Pop fleurs & Paris & $\begin{array}{l}\text { Fleuriste ateliers (plus d'actualité), } \\
\text { production dans les Hauts-de-France }\end{array}$ \\
\hline & Aquarelle.com & Hauts-de-Seine & Vente en ligne (bouquetterie) \\
\hline & Les Fleurs d'Ici & Paris & Vente en ligne (transmission florale) \\
\hline & Du pain et des roses & Paris & $\begin{array}{c}\text { Fleuriste évènementiel, association } \\
\text { d'insertion }\end{array}$ \\
\hline & Collectif de la fleur française & Paris & Association - réseau national \\
\hline
\end{tabular}

Figure 2 : Typologie des structures concernées par l'enquête de terrain*

* Des entretiens avec des personnes investies dans ces structures ont été menés entre décembre 2018 et avril 2020 en Ile-de-France ${ }^{6}$. La liste n'est pas exhaustive. Elle omet des structures dont les personnes ressources ont été rencontrées de manière moins formelle (via le Collectif de la fleur française notamment) et des structures pour lesquelles des informations disponibles en ligne ont servi à la rédaction de l'article (sites personnels des associations ou des entreprises).

Le présent article vise à retranscrire et questionner les facteurs de déterritorialisation et de reterritorialisation de la production de fleurs coupées en Ile-de-France. Dans un premier temps, les dynamiques ayant conduit à un déclin de la production sont étudiées. Le processus de déterritorialisation est-il réellement achevé ? Dans un deuxième temps, les facteurs potentiels de reterritorialisation sont abordés à travers l'émergence de modes de commercialisation dont l'origine et les pratiques culturales sont des arguments de vente majeurs, mais aussi à travers de nouvelles formes de production. Dans un troisième temps, des points pouvant enrayer le processus de reterritorialisation sont soulevés.

6 La plupart des entretiens ont eu lieu en Ile-de-France en avril 2019 et mars 2020 grâce au soutien financier du projet ANR RosesMonde. 


\section{Vers une production de fleurs coupées résiduelle en Ile-de-France}

À partir de la deuxième moitié du $\mathrm{XX}^{\mathrm{e}}$ siècle, la production de fleurs coupées francilienne diminue fortement et les fleurs importées sont de plus en plus nombreuses. Pour comprendre pourquoi, il faut changer d'échelle, car le phénomène est national. Toute la production française est en effet touchée par une réduction de la production qui commence après-guerre et s'intensifie à la fin du siècle. La culture des fleurs francilienne est aujourd'hui résiduelle.

\section{Déclin de la production française et francilienne au XXe siècle}

À partir des années 1970, une consommation de "masse " des fleurs coupées se développe. Le secteur de la distribution s'adapte. Les fleuristes font face, d'abord, à la concurrence de la grande distribution, qui intègre des fleurs coupées au rayon fruits et légumes, puis à celle de nouveaux fleuristes, précurseurs des franchises. Pour ces nouveaux distributeurs, il faut des fleurs standardisées et des volumes conséquents et constants que seuls les producteurs étrangers semblent être en capacité de fournir à cette époque ${ }^{7}$.

En effet, les producteurs français ont souvent des surfaces de production trop réduites et sont trop diversifiés pour avoir les volumes requis régulièrement pour répondre à la demande. Ils font, de plus, face à des difficultés qui limitent leurs investissements ${ }^{8}$. Les cultures sous serres, sont notamment fortement impactées par le choc pétrolier qui fait augmenter le coût de l'énergie? La pression foncière urbaine joue également un rôle important : des exploitations sont délocalisées voire abandonnées. Les producteurs proches de la retraite - relativement nombreux - ne trouvent pas de repreneurs et/ou n'en cherchent pas. Il est plus lucratif de revendre la terre. Cela est particulièrement vrai dans les zones de production autour des grandes villes comme Paris ${ }^{10}$. Dans ce contexte, les exploitants qui maintiennent leur production n'investissent pas faute de moyens ou par peur d'être délogés. Ils préfèrent se concentrer sur leur mise en marché (vente de leurs fleurs aux fleuristes ou aux consommateurs directement), quitte, pour certains, à entreprendre l'achat-revente dont la marge est plus lucrative. En théorie, cela démontre une certaine capacité à maîtriser le secteur de la commercialisation par les producteurs. Cependant, en

7 Christiane Perrin-Cook, L'horticulture ornementale française face à l'internationalisation des échanges, EHESS, Paris, Thèse de doctorat en économie, 1985, p. 49, 63-65, 69.

8 Christiane Perrin-Cook, "Quel avenir pour l'horticulture ornementale en France? ", Économie rurale, 161, 1984, p. 24-30.

9 Christiane Perrin-Cook, L'horticulture ornementale française face à l'internationalisation des échanges, EHESS, Paris, Thèse de doctorat en économie, 1985, p. 160.

10 Ségolène Darly, "Chapitre 2. Le partage de la terre agricole, entre conflits fonciers et projet collectif (l'exemple des domaines agricoles du Plateau briard), in Françoise Papy (éd.), Nouveaux rapports à la nature dans les campagnes, Éditions Quæ, 2012, p. 41-53. 
faisant cela, ils concurrencent les marchés de détail traditionnels et de gros et accentuent le déficit commercial car ils achètent des fleurs importées pour les revendre, facilitant ainsi la pénétration par les Hollandais du marché français. De plus, loin de regrouper les producteurs, ces pratiques les mettent davantage en concurrence. Dans le même temps, les exploitations hollandaises se sont, quant-à-elles, modernisées rendant la compétitivité des exploitations françaises de plus en plus difficile ${ }^{11}$.

Une dizaine d'années après le choc pétrolier et les premières ventes de fleurs en supermarchés, le constat est sans appel. Contrairement à d'autres cultures, comme les céréales, qui ont connu une période de développement importante après-guerre, la production de fleurs coupées a fortement décliné en Ile-de-France. Sur les 1000 hectares qui lui étaient consacrés en 1970, il en reste moins de 500 en $1988 .{ }^{12}$ L'expansion de Paris et de sa banlieue est très rapide ${ }^{13}$. Elle conduit les producteurs à se déplacer toujours plus loin de la petite vers la grande couronne ou à disparaître. De plus, l'ouverture des enchères florales hollandaises à la concurrence a encouragé la délocalisation de la production de fleurs dans la zone intertropicale dès les années 1980, accentuant encore davantage la concurrence entre producteurs franciliens et étrangers ${ }^{14}$. L'Ile-de-France compte tout de même 660 producteurs en $1985^{15}$.

Cependant, cette période est aussi celle des remises en question. Après le productivisme d'après-guerre qui a permis l'essor de la céréaliculture serait venu le temps du " post-productivisme " et des critiques de cette même céréaliculture. De nouveaux textes européens et nationaux viennent définir les nouvelles orientations agricoles en vue d'un développement durable. Ainsi, l'agriculture dans son ensemble est invitée à œuvrer pour les campagnes en devenant multifonctionnelle : nourricière, paysagère et respectueuse de la biodiversité. L'agriculture de proximité est fortement plébiscitée, au moins dans les discours, ce qui pourrait concourir à une reterritorialisation de la production ${ }^{16}$.

11 Christiane Perrin-Cook, L'horticulture ornementale française face à l'internationalisation des échanges, EHESS, Paris, Thèse de doctorat en économie, 1985, p. 17, 215-216.

12 Id., p. 23-25; Monique Poulot, «L'agriculture francilienne dans la seconde moitié du XXe siècle : vers un postproductivisme de proximité ? ", Pour, 205-206, 2010/2, p. 161-177.

13 Monique Poulot et Thérèse Rouyres, «La difficile évolution des campagnes franciliennes vers un espace multifonctionnel ", Hommes et Terres du Nord, 4, 2000, p. 253-260.

14 Léa Benoit, Bernard Calas, Sylvain Racaud, Olivier Ballesta, Lucie Drevet-Demettre, « Roses d'Afrique, roses du monde ", Géoconfluences, 2017

15 Christiane Perrin-Cook, L'horticulture ornementale française face à l'internationalisation des échanges, EHESS, Paris, Thèse de doctorat en économie, 1985, p. 23-25.

16 Collectif, "Une campagne dessinée par la ville. Une ville née de sa campagne », Pour, 2, 2010, p. 39-47 ; Monique Poulot, «L'agriculture francilienne dans la seconde moitié du XX siècle : vers un postproductivisme de proximité ? ", Pour, 2, 2010, p. 161-177. 
La production francilienne aujourd'hui : entre héritages et innovations

Aujourd'hui, de par leur faible nombre et leurs pratiques, les exploitations franciliennes semblent à la fois faire écho aux agricultures héritières de Donadieu qu'il perçoit comme des "vestiges en voie de disparition " des " ceintures urbaines" agricoles qui avaient pour mission "d'approvisionner les marchés urbains"; et aux agricultures innovantes "qui ne sont pas liées aux besoins alimentaires classiques " et rattachées à "l'évolution des modes de vie urbains $»^{17}$.

En 2018, la région ne compte plus que 20 exploitants, chiffre qui semble surestimé en 2020 et a diminué encore depuis - au moins une exploitation n'existe plus - et devrait diminuer encore.

Les exploitations sont situées dans la grande couronne parisienne, dans l'Essonne et en Seine-et-Marne, mais aussi dans d'autres départements franciliens de manière plus limitée ${ }^{18}$.

L'enquête invite à classer les exploitations en fonction des espèces qui y sont produites principalement : roses, fleurs à bulbes et fleurettes.

La production de roses a quasiment disparue. Les roses sont produites sous serres froides ou sous tunnel sur une poignée d'exploitations dans la zone historique au Sud-Est de Paris. La gamme variétale est large avec plus de 100 variétés sur certaines exploitations. Elle comprend majoritairement des roses de "niche " parfumées, issues de rosiers de jardin, branchues (Pierre de Ronsard, Yves Piaget). Cela a jusqu'ici permis aux producteurs de démarquer leurs roses sur le marché à côté des roses d'importation et du Sud de la France, qui sont pour l'essentiel plus "standardisées ", moins diversifiées et sans parfum, bien que certains rosiéristes franciliens déplorent aujourd'hui l'arrivée de roses parfumées de l'étranger. L'ancien schéma de complémentarité entre roses varoises d'hiver et roses franciliennes d'été semble en tout cas réinstauré. Ensuite, pour compléter leur gamme, les rosiéristes peuvent diversifier leur production sur l'année avec d'autres fleurs (à bulbes surtout) et/ou du feuillage; alors que d'autres pratiquent l'achat-revente.

Les fleurs à bulbes sont également produites par des producteurs dont c'est la spécialité ou qui les associent à des fleurettes. Â l'instar des roses, la production des fleurs à bulbes est saisonnière, mais la grande variété d'espèces permet d'échelonner la production : tulipes l'hiver et dahlias l'été, par exemple. Ces fleurs sont produites en plein air, sous serres froides et sous serres chauffées (davantage pour éviter le gel et avancer la saison). Elles sont également victimes de la concurrence étrangère et la culture de variétés différentes de celles importées leur assure une place sur le marché (tulipe perroquet, par exemple).

17 Pierre Donadieu, Campagnes urbaines, Actes Sud, 1998, p. 68-70.

18 FranceAgriMer, Observatoire des données structurelles des entreprises de production horticole et de la pépinière ornementales. Ile-de-France, 2019. 
Enfin, les fleurettes concernent toutes les autres fleurs produites en plein air (cosmos, nigelles, pois de senteurs). La définition de fleurettes n'est cependant pas très claire, certaines fleurs à bulbes étant parfois considérées comme appartenant à ce groupe (anémone, par exemple ${ }^{19}$ ). Ces fleurs sont moins concurrencées que les précédentes car elles sont moins facilement délocalisables et transportables. Elles sont également plus adaptées à un marché très sujet à la mode car ce sont souvent des annuelles. Ce sont elles qui semblent donc être les plus à même de résister au processus de déterritorialisation.

Un quatrième groupe pourrait venir compléter cette catégorisation : celui des feuillagistes et producteurs de sapins qui ont également une production de fleurs coupées (fleurettes généralement) et des producteurs vivriers qui viennent compléter leur offre mais n'apparaissent pas dans les statistiques des exploitations de fleurs coupées (pivoines d'arboriculteurs en mai-juin, par exemple).

Produire une gamme diversifiée de fleurs de saison contribuerait au maintien d'une certaine biodiversité et limiterait l'impact carbone des exploitations, ce qui permettrait de répondre en partie à la demande sociétale d'une agriculture respectueuse de l'environnement.

La pratique courante de l'agriculture raisonnée vient consolider ce phénomène. Elle peut être définie comme " un ensemble de pratiques de gestion d'exploitation visant à renforcer les impacts positifs de l'agriculture sur l'environnement et à en réduire les effets négatifs, sans remettre en cause la rentabilité économique des exploitations ${ }^{20}$. Cela inclut notamment la limitation de l'usage de produits phytosanitaires - qui sont de toute façon onéreux sans pour autant les interdire ${ }^{21}$.

La production de fleurs coupées en Ile-de-France a donc fortement décliné au $\mathrm{XX}^{\mathrm{e}}$ siècle. Le manque de compétitivité des exploitations face à la concurrence étrangère associée à la pression foncière due à l'étalement urbain en sont les causes. Pourtant, la consommation de fleurs coupées se démocratise à cette période et la demande augmente. La production française n'a pas su, ou pas pu, se saisir de cette opportunité. Les exploitations sont aujourd'hui trop peu nombreuses en Ile-de-France face au marché de consommation régional. L'urbanisation les a peu-à-peu repoussées dans la grande couronne où persiste une production diversifiée, essentiellement de saison et souvent conduite en agriculture raisonnée. Bien que deux exploitations aient été reprises ces dix dernières années, la production francilienne ne semble pas avoir totalement achevé son processus de déterritorialisation tant la concurrence étrangère et la pression foncière sont fortes.

19 D'après les mercuriales du RNM.

20 D'après Géoconfluences.

21 À savoir, certains produits sont cependant interdits en France alors qu'encore autorisés à l'étranger, provoquant une concurrence déloyale envers les producteurs français. 
Deux phénomènes viennent toutefois contrebalancer ce bilan et laissent penser qu'une reterritorialisation est possible. Tout d'abord, les producteurs continuent de maîtriser la mise en marché de leurs fleurs par la pratique des circuits-courts que l'État définit comme suit : «La notion de circuits-courts est utilisée pour valoriser un mode de vente limitant le nombre d'intermédiaires mais ne prévoit pas de notion de proximité physique (kilométrage ${ }^{22}$. Il s'agit en somme de réduire au maximum le nombre d'intermédiaires entre producteurs et consommateurs. Les exploitants sont de plus en plus soutenus en cela par l'émergence de structures de distribution franciliennes qui revendiquent une commercialisation alternative en faveur de la production régionale (voire nationale). Ensuite, des exploitations inédites ont été lancées ces dix dernières années en Ile-de-France venant grossir les rangs de la production.

\section{Vers une reterritorialisation de la production francilienne de fleurs coupées?}

\section{Une commercialisation alternative}

De plus en plus de fleuristes franciliens tentent d'acheter un maximum de fleurs locales et françaises, souvent de saison, tout en boycottant les fleurs étrangères et en communiquant auprès de leurs clients au sujet de leur démarche. Certains vont même jusqu'à inscrire l'origine de leurs fleurs en boutique (le café-fleuriste Désirée). Élément remarquable, dans la mesure où la mention de l'origine pour l'ornemental n'est pas obligatoire en France et souvent difficile à déterminer. Cette démarche est portée aussi bien par des artisans en boutique que par d'autres fleuristes qui pratiquent l'évènementiel ou organisent des ateliers. Parfois, elle est même associée à une vocation sociale, lorsque les fleuristes adhèrent à l'économie sociale et solidaire (Du Pain et des Roses) ou achètent leurs fleurs à des structures de production qui y adhèrent (Halage). Elle est possible grâce à deux avantages liés à la ville de Paris et à sa région.

Premièrement, nous pouvons supposer que la région parisienne constitue le premier bassin de consommation de fleurs coupées français. D’après une étude récente, la région parisienne est la région française qui engendre le plus de dépenses en végétaux d'intérieur et ce sont ces mêmes végétaux qui génèrent le plus de dépenses françaises $(59 \%)^{23}$. De plus, la mode est au "champêtre ", $\mathrm{au}$ " naturel ", au local. Les fleuristes qui appuient leur marketing sur la traçabilité (origine locale ou nationale) et la saisonnalité (fleurs les plus naturelles possibles) sont donc bien positionnés pour répondre à la demande.

22 D'après le portail de l'Économie, des Finances, de l'Action et des comptes publiques.

23 Kantar, Les achats de végétaux, arbres, plantes et fleurs des Français en 2018, 2019. 
Deuxièmement, le marché d'intérêt national de Rungis constitue une véritable plateforme logistique et confère à Paris et sa région une situation privilégiée pour la commercialisation des fleurs coupées.

Depuis 1969, ce marché international est en effet le principal lieu de mise en vente des fleurs franciliennes, mais aussi en provenance d'autres territoires français et étrangers. La plupart des producteurs franciliens commercialisent eux-mêmes leurs fleurs auprès des fleuristes sur le carreau, alors que d'autres ont créé une entreprise de gros et/ou d'import en parallèle. Cependant, le nombre de producteurs y a tellement diminué - passant de 300 à la fin des années 1980, à une douzaine aujourd'hui -, qu'il est possible de se demander si le carreau ne va pas disparaître. Producteurs, grossistes et importateurs continuent de se côtoyer et de se concurrencer avec une place de plus en plus réduite pour les premiers ${ }^{24}$. Les fleuristes tendent à déserter le marché à cause de la saturation du trafic routier francilien et de la fatigue, pour se tourner vers d'autres modes d'approvisionnement plus commodes comme les livraisons ${ }^{25}$. Les producteurs choisissent également d'éviter Rungis pour privilégier les circuits-courts hors marché, et ainsi éviter de travailler de nuit. Ils vendent par exemple leurs fleurs en direct (sur l'exploitation, sur des marchés de plein air), ou encore, aux fleuristes ou via des plateformes virtuelles.

La commercialisation de fleurs coupées via internet a émergé il y a une vingtaine d'années en France ${ }^{26}$. Cependant, ces cinq dernières années, à l'instar des fleuristes qui s'attachent à promouvoir les fleurs françaises et de saison, des sites internet d'un nouveau genre voient le jour en Ile-de-France (La Ruche qui dit Oui à la maison, Fleurs d'Ici, Monsieur marguerite). Les structures à l'origine de ces initiatives se différencient des précédentes par l'origine française de leurs fleurs et leur caractère saisonnier, véritables arguments de vente.

L'autre point commun entre ces initiatives est Paris et sa région. L'Ile-deFrance est propice au développement d'initiatives nouvelles, toujours par la présence de consommateurs et de la plateforme logistique, mais elle semble aussi avoir un rôle à jouer en termes de diffusion de modèles de commercialisation et de redistribution des fleurs sur le territoire.

Ces nouvelles structures de vente en ligne s'adaptent à l'offre des producteurs au fil des saisons. Elles leur assurent donc un nouveau débouché, sans pour autant remplacer leurs débouchés habituels.

24 Collectif, «Une campagne dessinée par la ville. Une ville née de sa campagne », Pour, 2, 2010, p. 39-47.

25 Christiane Perrin-Cook, L'horticulture ornementale française face à l'internationalisation des échanges, EHESS, Paris, Thèse de doctorat en économie, 1985, p. 44, 154 ; Annexes p. 38.

26 Caroline Maréchal, «Les artisans fleuristes, piliers du marché digital », Heure-digitale, Dossiers thématiques du Marché international de Rungis. 
Les pratiques de ces nouvelles structures de commercialisation ne sont cependant pas identiques, bien au contraire : il existe autant de manières de faire différentes que de structures (Figure 3). De plus, elles ne sont pas figées car ces structures évoluent, innovent en permanence.

\begin{tabular}{|c|c|c|c|c|}
\hline & $\begin{array}{c}\text { La Ruche qui dit } \\
\text { Oui }\end{array}$ & $\begin{array}{l}\text { La Ruche qui dit } \\
\text { Oui à la maison }\end{array}$ & Fleurs d'Ici & $\begin{array}{l}\text { Monsieur } \\
\text { marguerite }\end{array}$ \\
\hline Date de création & 2011 & 2018 & 2017 & 2016 \\
\hline Localisation & $\begin{array}{l}\text { Partout en France } \\
\text { (réseau de Ruches) }\end{array}$ & $\begin{array}{l}\text { Partout en Ile-de- } \\
\text { France (réseau de } \\
\text { Ruches) }\end{array}$ & Paris & Seine-Saint-Denis \\
\hline Principe & $\begin{array}{c}\text { Mise en relation } \\
\text { de producteurs et } \\
\text { consommateurs } \\
\text { locaux }\end{array}$ & $\begin{array}{c}\text { Mise en relation } \\
\text { de producteurs et } \\
\text { consommateurs } \\
\text { locaux en Ile-de- } \\
\text { France uniquement }\end{array}$ & $\begin{array}{l}\text { Mise en relation } \\
\text { de producteurs et } \\
\text { consommateurs } \\
\text { locaux via des } \\
\text { fleuristes locaux }\end{array}$ & $\begin{array}{l}\text { Mise en relation } \\
\text { de producteurs et } \\
\text { consommateurs } \\
\text { français }\end{array}$ \\
\hline $\begin{array}{c}\text { Produits } \\
\text { majoritaires }\end{array}$ & $\begin{array}{l}\text { Produits } \\
\text { alimentaires }\end{array}$ & $\begin{array}{l}\text { Produits } \\
\text { alimentaires }\end{array}$ & $\begin{array}{l}\text { Fleurs coupées } \\
\text { (bouquets) }\end{array}$ & $\begin{array}{c}\text { Fleurs coupées } \\
\text { (bottes et } \\
\text { bouquets) }\end{array}$ \\
\hline $\begin{array}{c}\text { Mode de } \\
\text { commercialisation } \\
\text { des fleurs coupées }\end{array}$ & $\begin{array}{c}\text { Retrait } \\
\text { hebdomadaire de } \\
\text { bottes de fleurs } \\
\text { par des particuliers } \\
\text { adhérents à la } \\
\text { Ruche après } \\
\text { commande }\end{array}$ & $\begin{array}{c}\text { Livraison } \\
\text { hebdomadaire } \\
\text { de bottes de } \\
\text { fleurs auprès } \\
\text { de particuliers } \\
\text { adhérents à la } \\
\text { Ruche après } \\
\text { commande }\end{array}$ & $\begin{array}{c}\text { Livraison } \\
\text { de bouquets } \\
\text { sous la forme } \\
\text { d'abonnement ou } \\
\text { à la commande } \\
\text { auprès } \\
\text { d'entreprises } \\
\text { et de particuliers }\end{array}$ & $\begin{array}{l}\text { Livraison de bottes } \\
\text { et de bouquets } \\
\text { sous la forme } \\
\text { d'abonnements } \\
\text { ou à la commande } \\
\text { auprès de } \\
\text { particuliers }\end{array}$ \\
\hline $\begin{array}{c}\text { Territoire de } \\
\text { commercialisation }\end{array}$ & $\begin{array}{c}\text { Le plus localement } \\
\text { possible }\end{array}$ & Ile-de-France & $\begin{array}{l}\text { Le plus } \\
\text { localement } \\
\text { possible }\end{array}$ & France entière \\
\hline $\begin{array}{l}\text { Intermédiaires } \\
\text { autres que la } \\
\text { plateforme }\end{array}$ & Non & Oui, livreurs & $\begin{array}{l}\text { Oui, fleuristes en } \\
\text { externe et livreurs }\end{array}$ & $\begin{array}{l}\text { Oui, fleuristes en } \\
\text { interne et livreurs }\end{array}$ \\
\hline $\begin{array}{l}\text { Niveau de mention } \\
\text { de l'origine sur le } \\
\text { site internet }\end{array}$ & $\begin{array}{l}\text { Nom du } \\
\text { producteur et } \\
\text { localisation }\end{array}$ & $\begin{array}{l}\text { Nom du } \\
\text { producteur et } \\
\text { localisation }\end{array}$ & $\begin{array}{l}\text { Origine locale, } \\
\text { marque de vente } \\
\text { "Fleurs d'Ici " }\end{array}$ & $\begin{array}{l}\text { Origine France, } \\
\text { nom du/des } \\
\text { producteurs et } \\
\text { localisation pour } \\
\text { les bottes }\end{array}$ \\
\hline $\begin{array}{c}\text { Rôle donné à } \\
\text { l'Ile-de-France par } \\
\text { l'intermédiaire de la } \\
\text { structure }\end{array}$ & $\begin{array}{l}\text { Renforcement de } \\
\text { l'agriculture de } \\
\text { proximité et des } \\
\text { liens entre espaces } \\
\text { ruraux et espaces } \\
\text { urbains }\end{array}$ & $\begin{array}{c}\text { Renforcement de } \\
\text { l'agriculture de } \\
\text { proximité et des } \\
\text { liens entre espaces } \\
\text { ruraux et espaces } \\
\text { urbains }\end{array}$ & $\begin{array}{c}\text { Diffusion d'un } \\
\text { nouveau modèle } \\
\text { de transmission } \\
\text { florale à travers la } \\
\text { France pour les } \\
\text { fleurs françaises }\end{array}$ & $\begin{array}{l}\text { Centralisation et } \\
\text { redistribution de } \\
\text { fleurs françaises }\end{array}$ \\
\hline
\end{tabular}

Figure 3 : Tableau regroupant des exemples de structures franciliennes commercialisant des fleurs françaises en ligne

Réalisation : Léa Benoit, 2020

Source : entretiens et sites internet des structures 
Cet engouement pour la valorisation des fleurs françaises se ressent également à travers la mention de l'origine France et la présence du logo Fleurs de France sur certains sites internet d'entreprises, qui vendaient jusqu' ici en partie des fleurs françaises sans forcément le mettre en avant. Ces mêmes entreprises cherchent à accroître leur offre nationale, mais se heurtent au manque de fleurs françaises, d'autant plus qu'elles ont souvent besoin de quantités importantes et standardisées. Ce sont elles qui reçoivent le plus de réticences de la part des producteurs, qui les jugent parfois trop opportunistes - surtout pour celles qui vendent aussi des fleurs d'importation - et non adaptées pour leurs fleurs. Elles pourraient cependant contribuer à une reterritorialisation de la production en tentant, par exemple, de participer à l'installation de nouveaux producteurs en les contractualisant.

Une reterritorialisation semble donc possible grâce à de nouvelles pratiques de commercialisation de la part de fleuristes et de nouvelles structures de ventes en ligne qui revendiquent l'origine françaises (voire plus locale) des fleurs et le respect des saisons. En jouant la carte de la traçabilité, elles répondent à une demande sociétale et communiquent sur la production francilienne/française de saison, recréant ainsi un lien entre producteurs et consommateurs sur un même territoire. Cela pourrait avoir un impact sur la demande en fleurs franciliennes/françaises et donc encourager l'installation de nouveaux producteurs. Paris et sa région semblent avoir un rôle moteur dans le développement de ces initiatives tant par la concentration de consommateurs que par le rôle de plateforme logistique.

La région semble également être propice au développement d'autres initiatives favorables à une reterritorialisation : des formes de production alternatives telles que des fermes urbaines et des chantiers d'insertion.

\section{Une production de fleurs coupées alternative}

Ces structures de production alternatives, très récentes, se distinguent des exploitations traditionnelles par leur statut, leur localisation souvent, mais surtout par leurs fonctions qui vont au-delà de la production et de la vente de fleurs.

Les fermes urbaines ont vu le jour dans le cadre des appels à projets Parisculteurs lancés par la mairie de Paris visant à re-végétaliser la capitale et à promouvoir l'agriculture urbaine. Parmi les lauréats, au moins un projet par an concerne la production de fleurs coupées : Plein air pour la saison un en 2018 et la Ferme florale urbaine pour la saison deux en 2019 dans Paris intramuros, et Murs à Fleurs dans la petite couronne, à Montreuil, pour la saison trois (en projet) ${ }^{27}$. Leur objectif premier est la production de fleurs coupées

27 Seules les deux premières fermes ont été étudiées pour l'enquête. 
par la revalorisation d'un terrain urbain délaissé et souvent non constructible, voire sur lequel il n'est pas possible de faire autre chose que pratiquer une agriculture non vivrière et non mécanisable. Les surfaces de production sont beaucoup plus réduites que pour les exploitations traditionnelles $\left(1000 \mathrm{~m}^{2}\right.$ environ) et la gamme produite plus diversifiée. Les techniques de production se rapprochent de celles de l'agriculture biologique - voire biodynamique non certifiée : production en plein air, de saison et sans produits chimiques. La commercialisation des fleurs est très locale : sur l'exploitation ou distribuées à des fleuristes parisiens à vélo.

Les chantiers d'insertion sont quant-à-eux situés aussi bien en zone urbaine qu'en zone rurale. Halage en Seine-Saint-Denis (production démarrée en 2018), et Fleurs de Cocagne, dans l'Essonne (2016) en sont deux exemples. À la différence des exploitations traditionnelles et des fermes urbaines, ils n'ont pas pour objectif principal la production et la commercialisation de fleurs, mais l'accompagnement économique et social de personnes éloignées de l'emploi et notamment de femmes. Ils sont d'ailleurs subventionnés par l'État en cela. Les fleurs sont un moyen d'atteindre cet objectif. Cependant, les surfaces cultivées se rapprochent de celles des exploitations traditionnelles (plus de $5000 \mathrm{~m}^{2}$ ), tout comme les types de production (en plein air, mais aussi sous tunnels et sous serres), ce qui en font des acteurs importants de la fleur coupée en Ile-de-France. Fleurs de Cocagne a d'ailleurs repris une exploitation sur laquelle étaient produites des roses en conventionnel. À noter toutefois, les pratiques de ces structures sont proches voire répondent à celles de l'agriculture biologique (Fleurs de Cocagne possède le label), ce que les producteurs traditionnels réfutent dans l'ensemble pour l'instant. Les modes de commercialisation sont également similaires : vente directe sur l'exploitation, livraisons aux fleuristes, vente en ligne.

Ces deux catégories ne sont cependant pas hermétiques.

Ainsi, un objectif social est affiché par les fermes urbaines qui peuvent, par exemple, travailler avec des employés en insertion ponctuellement ou projeter de réaliser des activités thérapeutiques avec les enfants d'un hôpital.

Ou encore, un chantier d'insertion peut également permettre de revaloriser un lieu pollué en zone urbaine.

Ce qui est frappant, c'est la multifonctionnalité de ces exploitations. Elle semble être la clef pour un ancrage territorial réussi et donc une reterritorialisation de la production dans des lieux où elle avait disparu. Les fermes urbaines et les chantiers sont multifonctionnels car en plus de venir accroître l'offre régionale en fleurs coupées et ainsi réduire la part des fleurs lointaines, ils ont des fonctions sociales, éducatives, environnementales et de recherche.

Fonctions sociales et éducatives, car ils encouragent l'insertion de personnes éloignées de l'emploi, mais aussi car ils peuvent entretenir des liens étroits avec les citoyens à proximité par la vente directe et l'organisation d'ateliers. Cette vocation est d'autant plus forte qu'elle concerne des citoyens peu habitués à voir 
des espaces de production agricoles au quotidien, et des produits - les fleurs dont la culture est réduite en France. La plupart des fermes urbaines et des chantiers d'insertion communiquent également via leur propre site internet, sur les réseaux sociaux et dans les médias. La vocation éducative se ressent également à travers la mise en réseau de ces structures avec les autres acteurs des fleurs coupées français via le Collectif de la Fleur française qui effectue un travail de partage et de communication.

Fonctions écosystémiques, car elles permettent de valoriser des lieux délaissés dans les interstices urbains, souvent pollués et exempts de végétation. Aussi, elles produisent une grande diversité de variétés de fleurs adaptées à la culture biologique en plein air et au climat francilien à forte amplitude thermique, constituant ainsi un nouvel espace pour les insectes. Ces fleurs, sont, dans la mesure du possible, différentes des fleurs produites à l'étranger et chez les producteurs franciliens traditionnels, ce qui contribue à accroître la biodiversité. Il y a là une opportunité à saisir en termes de création variétale.

Enfin, les fermes urbaines et les chantiers d'insertions remplissent une fonction de recherche, car ces structures ouvrent leurs portes à des obtenteurs et scientifiques prêts à tester de nouvelles variétés ou des produits non chimiques éligibles en agriculture biologique; ou font elles-mêmes un travail d'analyse de leurs propres pratiques. Elles sont lieux d'innovation à moindre coût.

Cette multifonctionnalité est remarquable car elle est non seulement reconnue, mais aussi voulue par d'autres acteurs que ceux qui la produisent (État, citoyens) et pourrait donc concourir à une reterritorialisation de la production de fleurs coupées. Comme le souligne Duvernoy, ce n'est pas la multifonctionnalité de l'agriculture qui est nouvelle, mais le fait que ses fonctions soient reconnues et cela impulse de nouvelles dynamiques ${ }^{28}$.

Ces nouvelles structures sont aussi cependant source d'inquiétude pour certains exploitants traditionnels, qui ne voient pas d'un bon œil la concurrence que ces structures subventionnées pourraient leur faire. Les quantités commercialisées aujourd'hui par les producteurs alternatifs semblent toutefois trop faibles pour que cela soit le cas, d'autant plus que la demande excède l'offre.

Malgré tous ces facteurs potentiels de reterritorialisation, plusieurs questions restent en suspens : comment assurer la viabilité des activités de production et de vente de fleurs coupées étudiées ? Quelle est la "bonne " distance de commercialisation des fleurs coupées ? Et plus largement, comment rendre attractif le métier de producteur de fleurs coupées dans un contexte de déprise agricole en France?

28 Isabelle Duvernoy, Françoise Jarrige, Paule Moustier, José Serrano, « Une agriculture multifonctionnelle dans le projet urbain : quelle reconnaissance, quelle gouvernance ?, Les Cahiers de la multifonctionnalité, 2005, p. 87-104. 


\begin{tabular}{|c|c|c|c|}
\hline & $\begin{array}{l}\text { Exploitations } \\
\text { traditionnelles }\end{array}$ & Fermes florales & Chantiers d'insertion \\
\hline Espace de production & Rural & Urbain & Rural ou urbain \\
\hline Taille de l'exploitation & $5000 \mathrm{~m}^{2}$ et plus & $1000 \mathrm{~m}^{2}$ & $5000 \mathrm{~m}^{2}$ et plus \\
\hline Nombre d'employés & + & - & ++ \\
\hline Aides de l'État & Aides de la PAC & $\begin{array}{c}\text { Bail obtenu via les } \\
\text { Parisculteurs }\end{array}$ & $\begin{array}{l}\text { Subvention du salaire } \\
\text { des salariés en insertion }\end{array}$ \\
\hline Type de production & $\begin{array}{l}\text { Plein air, serres froides, } \\
\text { serres chauffées } \\
\text { Production raisonnée }\end{array}$ & $\begin{array}{c}\text { Plein air } \\
\text { Production } \\
\text { biologique non } \\
\text { certifiée }\end{array}$ & $\begin{array}{l}\text { Plein air, serres froides, } \\
\text { serres chauffées } \\
\text { Production biologique } \\
\text { certifiée/non certifiée }\end{array}$ \\
\hline $\begin{array}{l}\text { Niveau de } \\
\text { diversification de la } \\
\text { production (suivant } \\
\text { le nombre de variétés } \\
\text { produites différentes) }\end{array}$ & $\begin{array}{c}\text { Roses, fleurs à bulbes, } \\
\text { fleurettes. Tendance à la } \\
\text { spécialisation dans l'une } \\
\text { de ses trois catégories }\end{array}$ & $\begin{array}{l}\text { Production très } \\
\text { diversifiée }\end{array}$ & $\begin{array}{c}\text { Production } \\
\text { moyennement à très } \\
\text { diversifiée }\end{array}$ \\
\hline Modes de vente & $\begin{array}{c}\text { Via Rungis, } \\
\text { Sur l'exploitation, en } \\
\text { direct aux fleuristes, via } \\
\text { des plateformes en ligne }\end{array}$ & $\begin{array}{l}\text { Sur l'exploitation, } \\
\text { en direct aux } \\
\text { fleuristes parisiens } \\
\text { à vélo }\end{array}$ & $\begin{array}{l}\text { Sur l'exploitation, en } \\
\text { direct aux fleuristes, via } \\
\text { des plateformes en ligne }\end{array}$ \\
\hline
\end{tabular}

Figure 4: Tableau de comparaison des producteurs traditionnels,

des fermes florales et des chantiers d'insertion

Réalisation : Léa Benoit, 2020. Source : entretiens

\section{Reterritorialisation en question(s)}

\section{La viabilité économique des nouvelles structures de production}

L'un des points phare qui ressort des entretiens est la fragilité aussi bien des fermes urbaines que des chantiers d'insertion. Qui dit nouveauté dit bien souvent instabilité. Plusieurs structures ne sont en effet pas encore viables et leurs gérants peinent à trouver le modèle économique idéal. Beaucoup se questionnent quant aux prix de ventes, aux subventions, ou encore aux labels.

Vendre, oui, mais à quel prix ? Le juste prix est difficile à définir pour les nouvelles structures de production. Faut-il se baser sur les prix de Rungis ou pas ? Faut-il vendre moins cher si l'on est plus proche des consommateurs que les exploitants traditionnels et subventionnés par l'État? Ou au contraire plus cher parce que la production a un impact social important ou suit les principes de l'agriculture biologique? Faut-il ajuster les prix en fonction des quartiers dans Paris? En somme, quelle valeur accorder aux fleurs franciliennes?

Ensuite, comment s'affranchir des aides étatiques ? Les nouvelles structures de production sont (ou l'ont été pour démarrer) dépendantes des aides de l'État. Les fermes florales ont bénéficié d'un bail via les Parisculteurs, alors que les chantiers d'insertion voient une partie des salaires financés par l'État. Les premières peuvent s'affranchir des aides qu'elles ont reçu. La question portera davantage sur la pérennité du lieu de production : le bail ne semble pas éternellement reconductible. 
Quant aux secondes, leur activité est intrinsèquement liée aux aides étatiques.

Enfin, faut-il répondre à un cahier des charges qui encadre la production (agriculture biologique) et/ou la vente (Fleurs de France) et si oui, comment en assumer le coût?

Cette même question se pose pour la plupart des producteurs traditionnels qui réfutent pour la plupart le label agriculture biologique - qui interdit les produits chimiques - et les labels et certifications en général contrairement à plusieurs de leurs homologues étrangers, voire français. Le coût des audits voire le simple fait de payer pour être certifié semble être un frein important. Enfin, dernier point, mais pas des moindres, les certifications et labels ne semblent pas utiles pour les producteurs franciliens car ils vendent leurs fleurs en circuits-courts auprès des fleuristes et des consommateurs et se disent donc à même de communiquer sur leurs pratiques culturales et l'origine des fleurs sans avoir besoin d'un logo.

\section{La «bonne » distance de commercialisation}

Faut-il privilégier le local ou le national ? Que veut-dire local ? à 10, 50, $100 \mathrm{~km}$ de distance ? Chaque structure de production et de vente semble avoir sa propre politique en la matière.

D'une part, les producteurs traditionnels, les fermes florales et les chantiers d'insertion, achètent une partie ou la totalité de leurs semences, bulbes et plants auprès de fournisseurs étrangers, preuve supplémentaire d'un manque de souveraineté française pour ce secteur. Elles revendent ensuite leurs fleurs dans un rayon pouvant aller de quelques kilomètres à plusieurs centaines de kilomètres.

D'autre part, les fleuristes et structures de vente en ligne qui ne vendent que des fleurs françaises s'approvisionnent aussi bien en Ile-de-France qu'ailleurs en France (dans le Var, par exemple). Pour la plupart, elles ne pourraient pas maintenir leurs ventes uniquement avec des fleurs franciliennes.

Comment justifier alors une vente ou un achat pour des fleurs qui parcourent plus de kilomètres que si elles allaient/arrivaient de l'étranger ? Il est en effet parfois reproché aux fleuristes d'acheter des fleurs du Sud de la France, plus éloignées de Paris que les fleurs néerlandaises, certes, davantage produites sous serres chauffées.

\section{Le maintien de l'agriculture}

Enfin, la pérennité du métier de producteur de fleurs coupées est remise en question par le manque d'attractivité et le manque de formation dans le domaine. 
D'une part, le métier présente plusieurs difficultés. Il est physique et attire peu les jeunes. Ensuite, il est menacé par la pression foncière qui incite davantage les exploitants à revendre leurs terres. Il est donc peu attractif. Et ce, d'autant plus que les petites exploitations spécialisées sont peu encouragées par l'État, qui est plus enclin à soutenir d'autres cultures plus " porteuses». Enfin, il faut ajouter à cela les difficultés d'embauche rencontrées par les gérants.

$\mathrm{Si}$, aucun repreneur ne se présente rapidement pour plusieurs exploitations dont les gérants sont près de la retraite, le nombre de producteurs devrait diminuer encore en Ile-de-France.

D'autre part, les nouveaux producteurs qui s'installent (avec le statut d'exploitant ou pas) n'ont souvent pas bénéficié de formation agricole classique (BAC et BTS agricole notamment) car ce sont souvent des personnes en reconversion et/ou elles jugent les formations de l'État inadaptées aux structures qu'elles veulent mettre en place (ferme florale). Plusieurs se sont formés ailleurs, dans une école spécialisée dans l'agriculture urbaine ou en ligne via un organisme étranger, ou encore en faisant des stages sur des exploitations.

Il en va de même pour les fleuristes, qui déplorent le peu d'intérêt accordé aux fleurs françaises dans le CAP fleuriste, voire le total décalage entre la formation et les besoins réels des fleuristes pour répondre aux nouvelles demandes des consommateurs.

Pour conclure, les dynamiques en cours autour des fleurs coupées en Ile-deFrance pourraient bien permettre une reterritorialisation de la production.

La déterritorialisation ne semble pas achevée, et pourtant, il existe des initiatives encourageantes en termes de production et de commercialisation. Les producteurs traditionnels produisent des fleurs de saison pour le marché local. Des modes de commercialisation alternatifs s'appuyant sur la traçabilité et l'usage d'internet viennent les soutenir. De nouvelles structures de production alternatives fondées sur la multifonctionnalité se développent.

Toutes ces initiatives sont d'autant plus encourageantes qu'elles se développent aussi partout en France. De nouvelles fermes florales ou café-fleuristes s'ouvrent ainsi un peu partout sur le territoire national.

Cependant, la nouveauté de ces initiatives est également source d'instabilité. Elles apportent des solutions mais aussi de nouvelles questions. La pérennité des exploitations et des structures de commercialisation n'est pas encore assurée, et ce, d'autant plus en contexte de pandémie.

\section{Léa Benoit}

UMR Les Afriques dans le Monde Université Bordeaux Montaigne lea.benoit@etu.u-bordeaux-montaigne.fr 
Léa Benoit est doctorante contractuelle en géographie à l'Université Bordeaux Montaigne sous la direction de Bernard Calas. Elle est rattachée à l'École Doctorale Montaigne-Humanités et à l'Unité mixte de recherche Les Afriques dans le Monde. Sa thèse porte sur la construction de la qualité dans une économie globalisée à travers la production et la commercialisation des fleurs coupées africaines, françaises et néerlandaises pour le marché français. Il s’agit de voir comment les différents acteurs du secteur des fleurs coupées se démarquent les uns des autres en luttant contre la concurrence et/ou en répondant aux nouvelles exigences des consommateurs et en quoi cela modifie les systèmes de commercialisation et de production. Elle a notamment publié un Mambo! (carnet de l'IFRA de Nairobi) à ce sujet pour les fleurs kenyanes et un article sur le Slow Flowers pour The Conversation.

\section{Résumé}

Paris semble être une ville motrice pour la promotion des fleurs françaises dans un contexte de forte concurrence internationale. Cet article étudie les dynamiques franciliennes qui pourraient laisser envisager une reterritorialisation de la production de fleurs coupées régionalement voire nationalement à travers trois tendances : une production francilienne et rurale de saison, des formes de commercialisation alternatives pour les fleurs françaises, une production alternative multifonctionnelle essentiellement urbaine.

\section{Mots-clés}

Géographie, fleurs, reterritorialisation, Paris, agriculture.

\section{Abstract}

Paris seems to be a driving force for promoting French flowers in a very competitive context. This paper examines the possibilities of developing a territorial approach in the production of cut flowers in Ile-de-France and in the whole France, with particular emphasis to the role of Paris through three ways: a rural and seasonal production around Paris, alternative marketing arrangements for French flowers, and a multifunctionnal and alternative production in an urban context essentially.

\section{Keywords}

Geography, flowers, reterritorialization, Paris, agriculture. 